\title{
Entanglement measure for general pure multipartite quantum states
}

\author{
Hoshang Heydar: $\otimes^{*}$ and Gunnar Björk \\ Department of Microelectronics and Information Technology, \\ Royal Institute of Technology (KTH), Electrum 229, SE-164 40 Kista, Sweden
}

(Dated: July 11, 2018)

\begin{abstract}
We propose an explicit formula for an entanglement measure of pure multipartite quantum states, then study a general pure tripartite state in detail, and at end we give some simple but illustrative examples on four-qubits and m-qubits states.
\end{abstract}

PACS numbers: 03.67.Mn, 42.50.Dv, 42.50.Hz, 42.65.Ky

\section{INTRODUCTION}

One of the unsolved problems of modern quantum theory is the quantification of multipartite state entanglement 1, 2]. This is a task that is directly linked to mathematics, such as linear algebra, geometry and functional analysis. The definition of separability and entanglement of a multipartite state was introduced in 3, 4] following the definition for bipartite states, given in 1989 by Werner 5]. Eventually, quantitative measures, such as the entanglement of formation and concurrence were formulated for bipartite systems [6]. In recent years, there have been attempts to find an entanglement measure for qubit-qudit states 7, 8, 9, 10 and for multipartite states, i.e., in [11, 12, 13, 14, 15, 16]. To exemplify, upper and lower bounds for the quantum relative entropy of entanglement of a multipartite systems in terms of the bipartite entanglements of formation, distillation, and quantum entropy of various subsystems are derived in 11]. Measures based on the Schmidt rank are proposed in [14] and on local unitary and filtering processes in [15]. Furthermore, in 17], a very useful tool to detect entanglement, called entanglement witness, is generalized to multipartite states. The tool is a consequence of the Hahn-Banach theorem which states that for any convex, compact, vector set $\mathcal{S}$, if $\rho \notin \mathcal{S}$, there exists a hyperplane that separates $\rho$ from $\mathcal{S}$. However, to find such an operator, even in case of tripartite state, is a formidable task. None-the-less, quite impressively, Acín et al. managed to construct a witness operator for a class of mixed tripartite states [18.

In a recent paper, Partovi proposes an entanglement measure based on generalized Schmidt-decompositions of a state [19]. In essence, his measure gives the difference between the minimum entropy of the separable state having the same statistical marginal distribution as the state being characterized, and the entropy of the state itself. To find the minimal difference, Partovi employs a successive Schmidt-decomposition of the state. The measure quantifies the logarithmic "quantumness" of a state irrespective of the type of entanglement is possesses.

*Electronic address:

hoshang@imit.kth.se
In this paper, we propose another measure of entanglement for arbitrary, pure multipartite states. Inspired by the work in 20], we give an explicit expression for such a functional. Our method is based on the joint relative-phase properties of a multipartite quantum system $\mathcal{Q}=\mathcal{Q}_{1} \otimes \mathcal{Q}_{2} \otimes \cdots \otimes \mathcal{Q}_{m}$ on a Hilbert space $\mathcal{H}_{\mathcal{Q}}=\mathcal{H}_{\mathcal{Q}_{1}} \otimes \mathcal{H}_{\mathcal{Q}_{2}} \otimes \cdots \otimes \mathcal{H}_{\mathcal{Q}_{m}}$ expressed by a positive operator value measure $(\mathrm{POVM}) \Delta_{\mathcal{Q}}$ on $\mathcal{H}_{\mathcal{Q}}$. The POVM is constructed by taking the $m$-fold tensor product of the subsystems' corresponding POVMs. We have already discussed, in detail, our degree of entanglement for a bipartite state in [21, 22], so here we will only give examples for multipartite states.

\section{ENTANGLEMENT FROM A RELATIVE-PHASE POVM}

A general and symmetric POVM in a single $N_{u^{-}}$ dimensional Hilbert space $\mathcal{H}_{\mathcal{Q}_{u}}$ is given by

$$
\hat{\Delta}_{Q_{u}}=\sum_{l_{u}}^{N_{u}} \sum_{k_{u}=1}^{N_{u}} e^{i \varphi_{k_{u}, l_{u}}}\left|k_{u}\right\rangle\left\langle l_{u}\right|
$$

where $\left|k_{u}\right\rangle$ are the basis vectors in $\mathcal{H}_{\mathcal{Q}_{u}}$ and

$$
\varphi_{k_{u}, l_{u}}=-\varphi_{l_{u}, k_{u}}\left(1-\delta_{k_{u} l_{u}}\right) .
$$

The POVM is a function of the $N_{u}\left(N_{u}-1\right) / 2$ relative phases $\left(\varphi_{1_{u}, 2_{u}}, \ldots, \varphi_{1_{u}, N_{u}}, \varphi_{2_{u}, 3_{u}}, \ldots, \varphi_{N_{u}-1, N_{u}}\right)$.

It is now possible to form a POVM of a multipartite system by simply forming the tensor product

$$
\begin{array}{r}
\hat{\Delta}_{\mathcal{Q}}\left(\varphi_{\mathcal{Q}_{1} ; k_{1}, l_{1}}, \ldots, \varphi_{\mathcal{Q}_{1} ; k_{m}, l_{m}}\right)= \\
\hat{\Delta}_{\mathcal{Q}_{1}}\left(\varphi_{\mathcal{Q}_{1} ; k_{1}, l_{1}}\right) \otimes \cdots \otimes \hat{\Delta}_{\mathcal{Q}_{m}}\left(\varphi_{\mathcal{Q}_{m} ; k_{m}, l_{m}}\right),
\end{array}
$$

where, e.g., $\varphi_{\mathcal{Q}_{1} ; k_{1}, l_{1}}$ is the set of POVM relative phases associated with subsystems $\mathcal{Q}_{1}$, for all $k_{1}, l_{1}=$ $1,2, \ldots, N_{1}$, where we need only to consider when $l_{1}>k_{1}$ due to (2). We can now recast this POVM, expressed in local properties, in terms of the relative-phase sums $\phi_{k_{1}, l_{1}, \ldots, k_{m}, l_{m}}=\sum_{u=1}^{m} \varphi_{k_{u}, l_{u}}$. Note that if, e.g., $l_{v}=k_{v}$, then the term $\varphi_{k_{v}}, k_{v}$ vanishes from the sum due to (2). From $\hat{\Delta}_{\mathcal{Q}}$ we can form an associated real function expressed in $\Pi_{u=1}^{m} N_{u}\left(N_{u}-1\right) / 2=M$ linearly independent 
relative-phase sums:

$$
\begin{aligned}
& \mathcal{P}\left(\phi_{k_{1}^{(1)}, l_{1}^{(1)}, \ldots, k_{m}^{(1)}, l_{m}^{(1)}}, \ldots, \phi_{k_{1}^{(M)}, l_{1}^{(M)}, \ldots, k_{m}^{(M)}, l_{m}^{(M)}}\right)= \\
& \operatorname{Tr}\left(\hat{\rho} \hat{\Delta}\left(\phi_{k_{1}^{(1)}, l_{1}^{(1)}, \ldots, k_{m}^{(1)}, l_{m}^{(1)}}, \ldots, \phi_{k_{1}^{(M)}, l_{1}^{(M)}, \ldots, k_{m}^{(M)}, l_{m}^{(M)}}\right)\right),(4)
\end{aligned}
$$

where $\hat{\rho}$ is the state density operator acting on the composite Hilbert space $\mathcal{H}_{\mathcal{Q}}$. Next, we define to what extent the density operator depends on the particular joint relative-phase $\operatorname{sum} \phi_{k_{1}^{(1)}, l_{1}^{(1)}, \ldots, k_{m}^{(1)}, l_{m}^{(1)}}$ e.g.,

$$
\begin{aligned}
& \gamma_{k_{1}^{(1)}, l_{1}^{(1)}, \ldots, k_{m}^{(1)}, l_{m}^{(1)}}= \\
& \mid \int_{2 \pi} d \phi_{k_{1}^{(1)}, l_{1}^{(1)}, \ldots, k_{m 1}^{(1)}, l_{m}^{(1)}} e^{-i \phi_{k_{1}^{(1)}, l_{1}^{(1)}, \ldots, k_{m}^{(1)}, l_{m}^{(1)}}} \\
& \mathcal{P}\left(\phi_{k_{1}^{(1)}, l_{1}^{(1)}, \ldots, k_{m}^{(1)}, l_{m}^{(1)}}, \ldots, \phi_{k_{1}^{(M)}, l_{1}^{(M)}, \ldots, k_{m}^{(M)}, l_{m}^{(M)}}\right) \mid
\end{aligned}
$$

where $\mathcal{P}$ must be expressed in the relative-phase sum parameter $\phi_{k_{1}^{(1)}, l_{1}^{(1)}, \ldots, k_{m}^{(1)}, l_{m}^{(1)}}$, but the particular choice of the remaining $M-1$ linearly independent relative-phase sum parameters is inconsequential for the absolute value of the integral. The coefficients $\gamma_{k_{u}, l_{u} \ldots, k_{v}, l_{v}}$ (where, here, and in the following, we will omit the superscript on the indices) are proportional to the Fourier components of the joint relative-phase distribution. Now, let us introduce the following index operator to connect the notation using the subsystem indices, and the notation using the joint-system index running from 1 to $N_{1} N_{2} \cdots N_{m}$ :

$$
\begin{aligned}
& \Pi\left(k_{1}, l_{1}, k_{2}, l_{2}, \cdots, k_{m}, l_{m}\right)=\left(k_{1}-1\right) N_{2} \cdots N_{m} \\
& +\left(k_{2}-1\right) N_{3} \cdots N_{m}+\cdots+\left(k_{m-1}-1\right) N_{m}+k_{m}, \\
& \left(l_{1}-1\right) N_{2} \cdots N_{m}+\left(l_{2}-1\right) N_{3} \cdots N_{m} \\
& +\cdots+\left(l_{m-1}-1\right) N_{m}+l_{m} .
\end{aligned}
$$

Note that the index operator generates two indices based on the set $\left\{k_{u}\right\}$ and $\left\{l_{u}\right\}$, respectively. Evaluating the Fourier components, one finds, not surprisingly, that $\gamma_{k_{1}, l_{1}, \ldots, k_{m}, l_{m}}=2 \pi\left|\rho_{\Pi\left(k_{1}, l_{1}, \ldots, k_{m}, l_{m}\right)}\right|$. That is, to each relative-phase sum there is an associated jointsystem density matrix coefficient. We now define an index permutation operator $\mathrm{P}_{j}$ operating on any function $f\left(k_{1}, l_{1}, \ldots, k_{m}, l_{m}\right)$ by

$$
\begin{array}{r}
\mathrm{P}_{j} f\left(k_{1}, l_{1}, \ldots, k_{j}, l_{j}, \ldots, k_{m}, l_{m}\right)= \\
f\left(k_{1}, l_{1}, \ldots, k_{j}, l_{j}, \ldots, k_{m}, l_{m}\right) \\
-f\left(k_{1}, l_{1}, \ldots, l_{j}, k_{j}, \ldots, k_{m}, l_{m}\right) .
\end{array}
$$

Using this operator we can generalize our earlier results for bipartite systems [21, 22]. We form an entanglement function by summing the absolute difference between pairwise relative-phase sums. The function is given by

$$
\begin{aligned}
& \Gamma(\hat{\rho})=\left(\left.\mathcal{N}_{2} \sum_{l_{1}>k_{1}}^{N_{1}} \sum_{k_{1}=1}^{N_{1}} \sum_{l_{2}>k_{2}}^{N_{2}} \sum_{k_{2}=1}^{N_{2}} \sum_{k_{3}=l_{3}=1}^{N_{3}} \ldots \sum_{k_{m}=l_{m}=1}^{N_{m}}\left|\mathrm{P}_{2}\right| \rho_{\Pi\left(k_{1}, l_{1}, k_{2}, l_{2}, \ldots, k_{m-1}, l_{m-1}, k_{m}, l_{m}\right)}\right|^{2}+\ldots\right. \\
& +\left.\mathcal{N}_{2} \sum_{l_{m-1}>k_{m-1}}^{N_{m-1}} \sum_{k_{m-1}=1}^{N_{m-1}} \sum_{l_{m}>k_{m} \mathcal{N}_{2}}^{N_{m}} \sum_{k_{m}=1}^{N_{m}} \sum_{k_{1}=l_{1}=1}^{N_{1}} \ldots \sum_{k_{m-2}=l_{m-2}=1}^{N_{m-2}}\left|\mathrm{P}_{m}\right| \rho_{\Pi\left(k_{1}, l_{1}, k_{2}, l_{2}, \ldots, k_{m-1}, l_{m-1}, k_{m}, l_{m}\right)}\right|^{2} \\
& +\mathcal{N}_{3} \sum_{l_{1}>k_{1}}^{N_{1}} \sum_{k_{1}=1}^{N_{1}} \sum_{l_{2}>k_{2}}^{N_{2}} \sum_{k_{2}=1}^{N_{2}} \sum_{l_{3}>k_{3}}^{N_{3}} \sum_{k_{3}=1}^{N_{3}} \sum_{i_{4}=j_{4}=1}^{N_{4}} \ldots \sum_{k_{m}=l_{m}=1}^{N_{m}}\left\{\left.\left|\mathrm{P}_{2}\right| \mathrm{P}_{3}\left|\rho_{\Pi\left(k_{1}, l_{1}, k_{2}, l_{2}, k_{3}, l_{3}, \ldots, k_{m-1}, l_{m-1}, k_{m}, l_{m}\right)}\right|\right|^{2} \mid\right\}+\ldots \\
& +\mathcal{N}_{3} \sum_{l_{m-2}>k_{m-2}}^{N_{m-2}} \sum_{k_{m-2}=1}^{N_{m-2}} \ldots \sum_{l_{m}>k_{m}}^{N_{m}} \sum_{k_{m}=1}^{N_{m}} \sum_{k_{1}=l_{1}=1}^{N_{1}} \ldots \sum_{i_{m-3}=j_{m-3}=1}^{N_{m-3}}\left\{\left|\mathrm{P}_{m-1}\right| \mathrm{P}_{m}\left|\rho_{\Pi\left(k_{1}, l_{1}, k_{2}, l_{2}, \ldots, k_{m-1}, l_{m-1}, k_{m}, l_{m}\right)}\right|^{2} \mid\right\} \\
& +\ldots+ \\
& +\mathcal{N}_{m-1} \sum_{l_{1}>k_{1}}^{N_{1}} \sum_{k_{1}=1}^{N_{1}} \ldots \sum_{l_{m-1}>k_{m-1}}^{N_{m-1}} \sum_{k_{m-1}=1}^{N_{m-1}} \sum_{k_{m}=l_{m}=1}^{N_{m}}\left\{\left.\left|\mathrm{P}_{2}\right| \mathrm{P}_{3}|\cdots| \mathrm{P}_{m-1}\left|\rho_{\Pi\left(k_{1}, l_{1}, k_{2}, l_{2}, \ldots, k_{m-2}, l_{m-2}, k_{m-1}, l_{m-1}, k_{m}, l_{m}\right)}\right|\right|^{2} \ldots|| \mid\right\} \\
& +\ldots+\mathcal{N}_{m-1} \sum_{l_{2}>k_{2}}^{N_{2}} \sum_{k_{2}=1}^{N_{2}} \ldots \sum_{l_{m}>k_{m}}^{N_{m}} \sum_{k_{m}=1}^{N_{m}} \sum_{k_{1}=l_{1}=1}^{N_{1}}\left\{\left|\mathrm{P}_{3}\right| \mathrm{P}_{4}|\cdots| P_{m}\left|\rho_{\Pi\left(k_{1}, l_{1}, k_{2}, l_{2}, k_{3}, l_{3}, \ldots, k_{m-2}, l_{m-2}, k_{m-1}, l_{m-1}, k_{m}, l_{m}\right)}\left\|^{2} \ldots \mid\right\|\right\}\right. \\
& \left.+\mathcal{N}_{m} \sum_{l_{1}>k_{1}}^{N_{1}} \sum_{k_{1}=1}^{N_{1}} \ldots \sum_{l_{m}>k_{m}}^{N_{m}} \sum_{k_{m}=1}^{N_{m}}\left\{\left.\left|\mathrm{P}_{2}\right| \mathrm{P}_{3}|\cdots| \mathrm{P}_{m}\left|\rho_{\Pi\left(k_{1}, l_{1}, k_{2}, l_{2}, \ldots, k_{m-1}, l_{m-1}, k_{m}, l_{m}\right)}\right|\right|^{2} \cdots|| \mid\right\}\right)^{\frac{1}{2}} .
\end{aligned}
$$

This is our central equation. It looks messy, but has a rather logical inner structure. The factors $\mathcal{N}_{u}$ 
are normalization factors, and they should not be confused with the space dimensions $N_{u}$. The first sums, where two of them are written explicitly (with normalization factors $\mathcal{N}_{2}$ ) on the right hand side of (8), only contributes the respective subsystem's bipartite entanglement. There are $\left(\begin{array}{c}m \\ 2\end{array}\right)=m(m-1) / 2$ ways to select two systems out of $m$ without respect to ordering. The two terms explicitly written above sums the bipartite entanglement contribution between systems $\mathcal{Q}_{1}, \mathcal{Q}_{2}$ and $\mathcal{Q}_{m-1}, \mathcal{Q}_{m}$, respectively. For the systems $\left\{\mathcal{Q}_{u}, \mathcal{Q}_{v}\right\}$, there are $N_{u}\left(N_{u}-1\right) N_{v}\left(N_{v}-1\right) / 4$ ways to select one each of the relative phases of system $\mathcal{Q}_{u}$ and $\mathcal{Q}_{v}$. Because the other system's coefficients can be chosen arbitrarily among the diagonals, there are $\prod_{j=1}^{m} N_{j} /\left(N_{u} N_{v}\right)$ number of relativephase sums and differences involving $k_{u}, l_{u}, k_{v}$ and $l_{v}$. Our permutation operator subtracts the relative-phase difference from the relative-phase sum, so by including all bipartite combinations, the bipartite entanglement of the joint system is taken care of. Next, we add the tripartite entanglement (contained in the sums with normalization factors $\left.\mathcal{N}_{3}\right)$. There are $\left(\begin{array}{c}m \\ 3\end{array}\right)$ tripartite combinations, and for every choice $\left\{\mathcal{Q}_{u}, \mathcal{Q}_{v}, \mathcal{Q}_{w}\right\}$, where $u<v<w$, there are $N_{u}\left(N_{u}-1\right) N_{v}\left(N_{v}-1\right) N_{w}\left(N_{w}-1\right) / 8$ combination of system relative phases. For each combination, we can sum all three relative phases, sum the first two and subtract the third, etc. To form differences of all combinations, we use both the permutation operators $\mathrm{P}_{v}$ and $\mathrm{P}_{w}$. Hence, we get $4=2^{3-1}$ contributions within the first curly bracket in (8), above. For each choice, the other systems indices can be chosen in $\prod_{j=1}^{m} N_{j} /\left(N_{u} N_{v} N_{w}\right)$ different ways. For the quadripartite contribution we proceed in the same way. For every choice $\left\{\mathcal{Q}_{u}, \mathcal{Q}_{v}, \mathcal{Q}_{w}, \mathcal{Q}_{z}\right\}$, where $u<v<w<z$, we use the permutation operators $\mathrm{P}_{v}, \mathrm{P}_{w}$, and $\mathrm{P}_{z}$. We get $8=2^{4-1}$ contributions inside the corresponding curly brackets, each being a sum of $\prod_{j=1}^{m} N_{j} /\left(N_{u} N_{v} N_{w} N_{z}\right)$ terms. The sum proceed in this fashion until the $m$-partite entanglement contributions are to be added. There is only one way $\left(\left(\begin{array}{c}m \\ m\end{array}\right)=1\right)$ to chose all subsystems, and we insert $m$ as index in our permutation operator. we use the permutation operators $\mathrm{P}_{2}, \mathrm{P}_{3}, \ldots, \mathrm{P}_{m}$. (We do not permute $k_{1}$ and $l_{1}$.) In all, we get $2^{m-1}$ terms inside the curly brackets of the last sum in (8), above. These terms represent all the possible relative-phase sums and differences between all the $m$-systems, so there are no further terms.

From our definitions, it is clear that for any product state

$$
\rho_{\Pi\left(k_{1}, l_{1}, k_{2}, l_{2}, \ldots, k_{m}, l_{m}\right)}=\rho_{k_{1}, l_{1}} \rho_{k_{2}, l_{2}} \cdots \rho_{k_{m}, l_{m}},
$$

where $\rho_{k_{u}, l_{u}}$ is the indicated density matrix coefficient of system $u$. In this case, one gets $\mathrm{P}_{u}\left|\rho_{\Pi\left(k_{1}, l_{1}, \ldots, k_{m}, l_{m}\right)}\right|=0$ for any $u$ and any set of indices $k_{1}, l_{1}, \ldots, k_{m}, l_{m}$. Hence, our entanglement function $\Gamma(\hat{\rho})=0$ for any tensor product of $m$ density operators. For entangled states, the function is not invariant to local unitary transformations. In analogy with our definitions for bipartite states, we define our measure of entanglement $\Gamma_{\text {sup }}$, where sup refers to the supremum of $\Gamma$ under all possible local unitary transformations.

Let us now write out and use (8) in a few explicit cases. The degree of entanglement for a $\mathcal{H}_{\mathcal{Q}_{1}} \otimes \mathcal{H}_{\mathcal{Q}_{2}}$ bipartite states is given by

$$
\begin{aligned}
& \Gamma(\hat{\rho})=\left(\mathcal{N}_{2} \sum_{l_{1}>k_{1}}^{N_{1}} \sum_{k_{1}=1}^{N_{1}} \sum_{l_{2}>k_{2}}^{N_{2}} \sum_{k_{2}=1}^{N_{2}} \| \rho_{\left(k_{1}-1\right) N_{2}+k_{2},\left(l_{1}-1\right) N_{2}+l_{2}} \mid\right. \\
&-\mid \rho_{\left(k_{1}-1\right)} N_{2}+l_{2},\left(l_{1}-1\right) N_{2}+k_{2} \\
&\left.\|_{\mathcal{Q}_{1} \mathcal{Q}_{2}}^{2}\right)^{\frac{1}{2}}
\end{aligned}
$$

This special case has already been discussed in detail in 21, 22], and we have shown that the equation coincides with the concurrence [6] for pure bipartite states in $2 \otimes 2$ (provided that one sets $\mathcal{N}_{2}=2$ ) and with generalized concurrence measures in $2 \otimes 3$ dimensions $[7,[8,9]$.

Note that our measure sums all the state's entanglement. That is, although, e.g., a state's bipartite entanglement between subsystems $\mathcal{Q}_{1}$ and $\mathcal{Q}_{2}$ cannot be used simultaneously neither with its bipartite entanglement between subsystems $\mathcal{Q}_{1}$ and $\mathcal{Q}_{3}$, nor, e.g., with its tripartite entanglement between subsystems $\mathcal{Q}_{1}, \mathcal{Q}_{2}$, and $\mathcal{Q}_{3}$, all contributions are added in our measure. That is, our measure characterizes the entanglement contained in a state, but in general the measure exceeds the usable entanglement. However, by looking at the various terms in the sum, the usable entanglement can be extracted as the measure is composed of sub-sums containing the bipartite $\mathcal{Q}_{1}$ and $\mathcal{Q}_{2}$ entanglement, the bipartite $\mathcal{Q}_{1}$ and $\mathcal{Q}_{3}$ entanglement, the tripartite $\mathcal{Q}_{1}, \mathcal{Q}_{2}$, and $\mathcal{Q}_{3}$ entanglement, etc., as can explicitly be seen in (11), below. Also note that our measure sums the possible cooperative entanglement. That is, if some subsystems are ignored, or the information contained in a subsystem is lost, then the ensuing state's entanglement is in general lower than what our measure predicts. We shall give a concrete example of this in Sec. IV] below.

\section{TRIPARTITE ENTANGLEMENT}

The degree of entanglement for a $\mathcal{H}_{\mathcal{Q}_{1}} \otimes \mathcal{H}_{\mathcal{Q}_{2}} \otimes \mathcal{H}_{\mathcal{Q}_{3}}$ tripartite state is given by 


$$
\begin{aligned}
\Gamma(\hat{\rho})= & \left(\mathcal { N } _ { 2 } \left[\sum_{l_{1}>k_{1}}^{N_{1}} \sum_{k_{1}=1}^{N_{1}} \sum_{l_{2}>k_{2}}^{N_{2}} \sum_{k_{2}=1}^{N_{2}} \sum_{k_{3}=l_{3}=1}^{N_{3}}|| \rho_{\Pi\left(k_{1}, l_{1}, k_{2}, l_{2}, k_{3}, l_{3}\right)}|-| \rho_{\Pi\left(k_{1}, l_{1}, l_{2}, k_{2}, k_{3}, l_{3}\right)} \|_{\mathcal{Q}_{1} \mathcal{Q}_{2}}^{2}\right.\right. \\
& +\sum_{l_{1}>k_{1}}^{N_{1}} \sum_{k_{1}=1}^{N_{1}} \sum_{l_{3}>k_{3}}^{N_{3}} \sum_{k_{3}=1}^{N_{3}} \sum_{k_{2}=l_{2}=1}^{N_{2}}|| \rho_{\Pi\left(k_{1}, l_{1}, k_{2}, l_{2}, k_{3}, l_{3}\right)}|-| \rho_{\Pi\left(k_{1}, l_{1}, k_{2}, l_{2}, l_{3}, k_{3}\right)} \|_{\mathcal{Q}_{1} \mathcal{Q}_{3}}^{2} \\
& \left.+\sum_{l_{2}>k_{2}}^{N_{2}} \sum_{k_{2}=1}^{N_{2}} \sum_{l_{3}>k_{3}}^{N_{3}} \sum_{k_{3}=1}^{N_{3}} \sum_{k_{1}=l_{1}=1}^{N_{1}}|| \rho_{\Pi\left(k_{1}, l_{1}, k_{2}, l_{2}, k_{3}, l_{3}\right)}|-| \rho_{\Pi\left(k_{1}, l_{1}, k_{2}, l_{2}, l_{3}, k_{3}\right)} \|_{\mathcal{Q}_{2} \mathcal{Q}_{3}}^{2}\right] \\
& +\mathcal{N}_{3} \sum_{l_{1}>k_{1}}^{N_{1}} \sum_{k_{1}=1}^{N_{1}} \sum_{l_{2}>k_{2}}^{N_{2}} \sum_{k_{2}=1}^{N_{2}} \sum_{l_{3}>k_{3}}^{N_{3}} \sum_{k_{3}=1}^{N_{3}}\left\{||\left|\rho_{\Pi\left(k_{1}, l_{1}, k_{2}, l_{2}, k_{3}, l_{3}\right)}\right|-\mid \rho_{\Pi\left(k_{1}, l_{1}, k_{2}, l_{2}, l_{3}, k_{3}\right)} \|_{\mathcal{Q}_{1} \mathcal{Q}_{2} \mathcal{Q}_{3}}^{2}\right. \\
& \left.\left.-|| \rho_{\Pi\left(k_{1}, l_{1}, l_{2}, k_{2}, k_{3}, l_{3}\right)}|-| \rho_{\Pi\left(k_{1}, l_{1}, l_{2}, k_{2}, l_{3}, k_{3}\right)} \|_{\mathcal{Q}_{1} \mathcal{Q}_{2} \mathcal{Q}_{3}}^{2} \mid\right\}\right)^{\frac{1}{2}} .
\end{aligned}
$$

Let us now give two concrete examples of this measure for some three-qubit states. In the three-qubit space there exist two classes of states, inequivalent under local operations and classical communication (LOCC), called $\left|\Psi_{\mathrm{GHz}}\right\rangle$ and $\left|\Psi_{\mathrm{W}}\right\rangle$ states. They are, e.g., $\left|\Psi_{\mathrm{GHz}}\right\rangle=$ $(|000\rangle+|111\rangle) / \sqrt{2}$ and $\left|\Psi_{\mathrm{W}}\right\rangle=(|001\rangle+|010\rangle+|100\rangle) / \sqrt{3}$. For these states, we have

$$
\Gamma\left(\hat{\rho}_{\mathrm{GHZ}}\right)=\left(\mathcal{N}_{3}\left|\rho_{1,8}\right|_{\mathcal{Q}_{1} \mathcal{Q}_{2} \mathcal{Q}_{3}}^{2}\right)^{\frac{1}{2}}=\sqrt{\frac{\mathcal{N}_{3}}{4}},
$$

and

$$
\begin{aligned}
\Gamma\left(\hat{\rho}_{\mathrm{W}}\right) & =\left(\mathcal{N}_{2}\left(\left|\rho_{3,5}\right|_{\mathcal{Q}_{1} \mathcal{Q}_{2}}^{2}+\left|\rho_{2,5}\right|_{\mathcal{Q}_{1} \mathcal{Q}_{3}}^{2}+\left|\rho_{2,3}\right|_{\mathcal{Q}_{2} \mathcal{Q}_{3}}^{2}\right)\right)^{\frac{1}{2}} \\
& =\sqrt{\frac{\mathcal{N}_{2}}{3}} .
\end{aligned}
$$

Here, we see that the normalization factors must be retained (or, possibly be chosen with particular relative weights) in order for the entanglement measure to make sense for general states. Because the GHZ- and the Wstates belong to different equivalence classes, their relative entanglement weights are not obvious. This issue is tied to the, still open, question about minimum reversible entanglement generating sets [2, 12, 13, 18, 23].

\section{BEYOND TREE-PARTITE QUBIT ENTANGLEMENT}

Next, let us look at an interesting four-qubit state $\left|\Psi_{1}\right\rangle=(|0,0,0,1\rangle+|0,1,0,0\rangle+|1,0,1,0\rangle+|1,1,1,1\rangle) / 2$. Our measure of entanglement of this state is

$$
\begin{aligned}
\Gamma\left(\Psi_{1}\right)= & \left(\mathcal{N}_{2}\left(\left|\rho_{2,5}\right|_{\mathcal{Q}_{2} \mathcal{Q}_{4}}^{2}+\left|\rho_{11,16}\right|_{\mathcal{Q}_{2} \mathcal{Q}_{4}}^{2}\right)\right. \\
& +\mathcal{N}_{3}\left(\left|\rho_{2,16}\right|_{\mathcal{Q}_{1} \mathcal{Q}_{2} \mathcal{Q}_{3}}^{2}+\left|\rho_{5,11}\right|_{\mathcal{Q}_{1} \mathcal{Q}_{2} \mathcal{Q}_{3}}^{2}\right. \\
& \left.\left.+\left|\rho_{2,11}\right|_{\mathcal{Q}_{1} \mathcal{Q}_{3} \mathcal{Q}_{4}}^{2}+\left|\rho_{5,16}\right|_{\mathcal{Q}_{1} \mathcal{Q}_{3} \mathcal{Q}_{4}}^{2}\right)\right)^{\frac{1}{2}} \\
= & \left(\frac{\mathcal{N}_{2}}{8}+\frac{\mathcal{N}_{3}}{4}\right)^{\frac{1}{2}} .
\end{aligned}
$$

The state has both bipartite and tripartite entanglement. In order to use the bipartite entanglement the parties possessing the different qubit subsystems must cooperate. If, e.g., qubit 1 and 3 are measured in the standard basis, the result is either two zeros or two ones. If this result is communicated to the parties holding qubit 2 and 4, (that is, we perform a LOCC, optimal for bringing out the bipartite entanglement) the remaining two-qubit state will be in (a known) pure EPR-state. If, on the other hand, if we simply trace out subsystems $\mathcal{Q}_{1}$ and $\mathcal{Q}_{3}$, (or measure qubit 1 and 3 but keep the result secret), then the remaining state is in an an equal mixture of the EPR-states, and this state is directly separable. This means that if the different parties do not cooperate, the state's bipartite entanglement in subspace 1 and 3 vanishes.

The entanglement of the state $\left|\Psi_{2}\right\rangle=(|0,1,1,0\rangle+$ $|1,0,0,1\rangle+|0,1,1,1\rangle+|1,0,0,0\rangle) / 2$, on the other hand, is given by

$$
\Gamma\left(\Psi_{2}\right)=\left(\mathcal{N}_{3}\left|\rho_{7,9}\right|_{\mathcal{Q}_{1} \mathcal{Q}_{2} \mathcal{Q}_{3}}^{2}\right)^{\frac{1}{2}}=\sqrt{\frac{\mathcal{N}_{3}}{4}}
$$

That is, the state has only tripartite entanglement and no bipartite entanglement. To arrive at the result, we note that a unitary transformation $\hat{U}_{4}$ local to $\mathcal{Q}_{4}$ can transform the state into, e.g., $\hat{U}_{4}\left|\Psi_{2}\right\rangle=(|0,1,1\rangle+|1,0,0\rangle \otimes$ $|0\rangle) / \sqrt{2}$ for which one finds the supremum of $\Gamma$. In this case, the state's entanglement is the same whether or not the person in possession of qubit 4 cooperates or not.

As a last example, consider a a $m$-qubit density operator given by a mixture of the two orthogonal, so called, $m$-Cat states

$$
\left|\Psi_{\text {Cat }}\right\rangle=\frac{1}{\sqrt{2}}\left(\left|0_{1}, 0_{2}, \ldots, 0_{m}\right\rangle+\left|1_{1}, 1_{2}, \ldots, 1_{m}\right\rangle\right)
$$


. Then, our degree of entanglement gives

$$
\begin{aligned}
\Gamma\left(\Psi_{\text {Cat }}\right) & =\left(\mathcal{N}_{m}\left|\rho_{1,2^{m}}\right|_{\mathcal{Q}_{1} \mathcal{Q}_{2} \cdots \mathcal{Q}_{m}}^{2}\right)^{\frac{1}{2}} \\
& =\left(\frac{\mathcal{N}_{m}}{4}\right)^{\frac{1}{2}} .
\end{aligned}
$$

\section{CONCLUSIONS}

In conclusion, we have proposed an entanglement measure for pure multipartite quantum states. The measure directly detects product states (it is zero for such states), and quantifies the entanglement of any pure state up to the bipartite, tripartite, ..., m-partite normalization coefficients. Since it is not possible to use, nor convert, the entanglement in states with incompatible entanglement classes such as GHZ- and W-states into each other, it may not be meaningful to specify the coefficients relative to each other. Rather, from an operational point of view, it seems more meaningful to specify each type of entanglement separately, e.g. in a system composed of four subsystems $\mathcal{Q}_{1}, \mathcal{Q}_{2}, \mathcal{Q}_{3}$, and $\mathcal{Q}_{4}$, it is meaningful to discuss, separately, the bipartite entanglement between, e.g., systems $\mathcal{Q}_{1}$ and $\mathcal{Q}_{2}$, and $\mathcal{Q}_{1}$ and $\mathcal{Q}_{4}$. We do not see how the bipartite entanglement, in an operational sense, could (or should) be compared to, e.g., the tripartite entanglement between $\mathcal{Q}_{1}, \mathcal{Q}_{2}$, and $\mathcal{Q}_{4}$. Our measure sums all contributions to quantify the state's entire entanglement, but, as just indicated, from an operational viewpoint, it is rather the the sum's various contributions that have a well defined operational meaning. This is in contrast to, e.g., Partovi's measure [19], that is a minimum entropic distance measure between the state and a separable state with the same statistical marginal distributions. In this sense, Hossein Partovi's measure is a better measure of the "quantumness" of the state, while our measure emphasize the state's usefulness as a quantum information carrier.

\section{Acknowledgments}

We wish to thank Professor L. L. Sánchez-Soto for inspiration and useful discussions. This work was supported by the Swedish Research Council (VR) and the Swedish Foundation for Strategic Research (SSF).
[1] M. Lewenstein, D. Bruß, J. I. Cirac, B. Kraus, M. Kuś, J. Samsonowicz, A. Sanpera, and R. Tarrach, J. Mod. Opt. 47, 2841 (2000).

[2] W. Dür, J. I. Cirac, and R. Tarrach, Phys. Rev. Lett. 83, 3562 (1999).

[3] V. Vedral, M. B. Plenio, M. A. Rippin, and P. L. Knight, Phys. Rev. Lett. 78, 2275 (1997).

[4] V. Vedral, M. B. Plenio, K. Jacobs, and P. L. Knight, Phys. Rev. A 58, 883 (1998).

[5] R. F. Werner, Phys. Rev. A 40, 4277 (1989).

[6] W. K. Wootters, Phys. Rev. Lett. 80, 2245 (1998).

[7] P. Rungta, V. Bužek, C. M. Caves, M. Hillery, and G. J. Milburn, Phys. Rev. A 64, 042315 (2001).

[8] S. Albeverio and S. M. Fei, J. Opt. B: Quantum Semiclass. Opt. 3, 223 (2001).

[9] K. Audenaert, F. Verstraete, and B. De Moor, Phys. Rev. A 64, 012316 (2001).

[10] E. Gerjuoy, Phys. Rev. A 67, 052308 (2003).

[11] M. B. Plenio and V. Vedral, J. Phys. A: Math. Gen. 34, 6997 (2001).

[12] C. H. Bennett, S. Popescu, D. Rohrlich, J. Smolin, and A. V. Thapliyal, Phys. Rev. A 63, 012307 (2001).

[13] W. Dür, G. Vidal, and J. I. Cirac, Phys. Rev. A 62,
062314 (2000).

[14] J. Eisert and H. J. Briegel, Phys. Rev. A 63, 022306 (2000).

[15] F. Verstraete, J. Dehaene, and B. De Moor, Phys. Rev. A 68, 012103 (2003).

[16] K. Eckert, O. Gühne, F. Hulpke, P. Hyllus, J. Korbicz, J. Mompart, D. Bruß, M. Lewenstein, and A. Sanpera, in Quantum Information Processing, edited by G. Leuchs and T. Beth (Wiley-VCH, Berlin 2003).

[17] M. Horodecki, P. Horodecki, and R. Horodecki, Phys. Lett. A 283, 1 (2001).

[18] A. Acín, D. Bruß, M. Lewenstein, and A. Sanpera, Phys. Rev. Lett. 87, 040401 (2001).

[19] M. H. Partovi, Phys. Rev. Lett. 92, 077904 (2004).

[20] L. L. Sánchez-Soto, J. Delgado, A. B. Klimov, and G. Björk, Phys. Rev. A 66, 042112 (2002).

[21] H. Heydari, G. Björk, and L. L. Sánchez-Soto, Phys. Rev. A 68, 062314 (2003).

[22] H. Heydari and G. Björk, e-print quant-ph/0401128 v1.

[23] E. F. Galvão, M. B. Plenio, and S. Virmani, J. Phys. A: Math. Gen. 33, 8809 (2000). 